\title{
Analysis of Separation Control Authority of DBD Plasma Actuator using Hot-Film Sensor Array
}

\author{
A. Daliri ${ }^{1}$, M. J. Maghrebi ${ }^{1 \dagger}$ and M. R. Soltani ${ }^{2,3}$ \\ ${ }^{I}$ Mechanical Engineering Faculty, Ferdowsi University of Mashhad, Mashhad, Iran \\ ${ }^{2}$ William E. Boeing Department of Aeronautics and Astronautics, Washington University, Seattle, \\ Washington, USA \\ ${ }^{3}$ Department of Aerospace Engineering, Sharif University of Technology, Tehran, Iran
}

†Corresponding Author Email: mjmaghrebi@um.ac.ir

(Received November 17, 2020; accepted February 19, 2021)

\begin{abstract}
The boundary-layer control authority of a DBD plasma actuator using surface mounted hot-film sensors is evaluated. Wind tunnel experiments on a wind-turbine blade section were established at a Reynolds number of $0.27 \times 10^{6}$. Aerodynamic performance of the wind-turbine blade section for both plasma-ON and plasmaOFF modes are evaluated using measurements made by both surface pressure and wake survey behind the model. Two distinct boundary-layer states are recognized. A state which occurs at the onset and in proximity of the deep stall, which is affected by the low-frequency instabilities of the separated flow. In this case, the steady actuation of plasma imparts local momentum on the nearby flow, eliminating the instabilities, hence, reattaching the detached flow. The other state happens beyond the static stall angle of attack of the airfoil where the flow over the suction side of the airfoil is fully separated and coexistence of both the leading edge and the trailing edge shear-layer instabilities and natural trailing edge vortex shedding is the underlying mechanism. In this case, although the plasma actuator eliminates the instabilities, to some extent, but the corresponding momentum injection is not efficient to stabilize and reattach the flow.
\end{abstract}

Keywords: DBD plasma actuator; Experimental aerodynamics; Wind tunnel testing; Flow control.

\section{NOMENCLATURE}

$\begin{array}{ll}c & \text { airfoil chord length } \\ \mathrm{Cp}_{\mathrm{p}} & \text { pressure coefficient } \\ \mathrm{C}_{\mathrm{L}} & \text { lift coefficient } \\ \mathrm{C}_{\mathrm{D}} & \text { drag coefficient } \\ E & \text { hot-film output voltage } \\ f & \text { frequency }\end{array}$

\section{INTRODUCTION}

All boundary-layer control methods adjust flow behavior near the surface by three main strategies i.e. prevent/provoke separation, delay/advance transition and suppress/enhance turbulence. These strategies lead to drag reduction, lift augmentation, mixing enhancement, heat transfer improvement and flow induced noise suppression (Gad-el-Hak 1989). The presence of both wall friction and adverse pressure gradient reduces the momentum of the fluid particles in a boundary-layer. In terms of the energy principles, the kinetic energy gained at the expense of the potential energy in the favorablepressure-gradient region is depleted by the viscous effects within the boundary-layer. In the adverse-

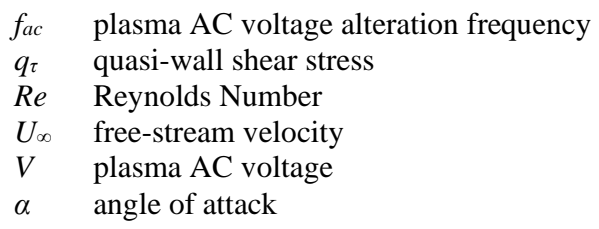

pressure-gradient region, the remaining kinetic energy is eventually exhausted and the motion of fluid particles close to the wall is pulled in. At this moment the viscous layer breaks away from the bounding surface and the streamline nearest to the wall leaves the body. The boundary-layer, in this point, is said to be separated. Due to the thick rotational flow region as well as the increased normal velocity component, large energy losses take place in the separation zone (Gad-el-Hak 1989). The performance of many practical devices is hampered by the location and extent of the separation zone. For example, the circulation and consequently, the lift of a wind-turbine blade section at high angles of attack could be enhanced, and its pressure drag would be reduced if the 
separation is postponed. The flow separation leads to the stall as the angle of attack of an airfoil increases that imposes considerable aerodynamic performance penalties. For the thick blade sections of the wind-turbine blades, the trailing-edge separation progressively moves towards the leading-edge with an increase in the angle of attack, and the lift coefficient of the section decreases significantly. The boundary-layer control commonly postpones separation by adding kinetic energy to the flow particles near the surface.

As the result of the growing instabilities in the laminar boundary-layer, transition from laminar flow to turbulence occurs. Further details on the mechanisms and paths of transition can be found in a review by Saric et al. (2001). In the turbulent region, mixing enhances and both shear stress and heat transfer increase, which leads to the increase of skin friction drag for the wall bounded flow; flow over an airfoil. The flow-induced noise results from the pressure fluctuations in the turbulent boundarylayer which is not inherently existent in the laminar boundary-layer. Maintaining a laminar boundarylayer on bodies subject to an external flow (e.g. wind-turbine blade section) is of great importance in the field of the boundary-layer control.

Various actuators can be used for the flow control applications. Further details on the mechanism and on the features of these actuators are addressed in a review by Cattafesta et al. (2011). The plasma actuators are among the main category of flow control actuators. A particular type of the nonthermal plasma actuators that has gained wide use is based on the dielectric-barrier-discharge (DBD) mechanism that has desirable features for use in air at atmospheric pressures (Cattafesta et al. 2011). These actuators work based on the formation of a low-temperature plasma between a pair of asymmetric electrodes by application of a highvoltage AC signal across the electrodes. Consequently, the air molecules surrounding the electrodes are ionized, and a body force parallel to the wall (known as the ionic wind) is induced by the actuator. The plasma actuators can operate in two different modes of steady and unsteady actuation. The steady actuation locally imparts momentum to the nearby flow and causes significant modification of the stability properties in the boundary-layer. On the other hand, unsteady forcing of the plasma actuator attenuates the natural instabilities of the flow by generating large coherent vortical structures (Abdollahzadeh et al. 2018)

Numerous experimental and numerical investigations were performed on the flow control with the plasma actuators. Many successful applications of the plasma actuators are reported in the literature. Some of them are: flow separation control on low-pressure turbine blades (Junhui et al. 2006a; Junhui et al. 2006b; Rizzetta et al. 2007), flow separation control on swept wing (Guangyin et al. 2015), lift force enhancement and drag reduction (Corke et al. 2007; Post et al. 2004a; Patel et al. 2007; Sosa et al. 2006), airfoil leading-edge separation control (Tathiri et al. 2016, Post et al. 2004; Orlov et al. 2007; Post et al. 2004b) shedding frequency alteration behind a circular cylinder (McLaughlin et al. 2004), separation bubble control on an iced airfoil (Pouryoussefi et al. 2016), stall control on a pitching airfoil (Woo et al. 2013), dynamic stall vortex control (Post et al. 2006; Lombardi et al. 2013) and wind-turbine blade separation control (Greenblatt et al. 2012; Jukes et al. 2015), etc.

Recently, measurements of plasma actuators bodyforce and induced flow have been extensively performed. The aerodynamic forces generated by a single dielectric-barrier-discharge (SDBD) plasma actuator were measured using total pressure boundary-layer probe, and an innovative pendulum force balance (Porter et al. 2007; Enloe et al. 2008a). The application of an accurate electronic force balance to measure the plasma induced force is demonstrated too (Hoskinson et al. 2008). Other direct methods such as electrical voltage or light emission measurement (Enloe et al. 2006; Enloe et al. 2008b) and smoke flow visualizations (McLaughlin et al. 2004; Roth et al. 2000) were also used. The effects of the plasma actuator on the fluid in the vicinity of the electrodes are further measured by means of hot-wire probe (Duchmann et al. 2014; Jukes et al. 2006), wall pressure measurement (Labergue et al. 2014), particle image velocimetry (PIV) (Labergue et al. 2014; Balcon et al. 2009; Kriegseis et al. 2012; Forte et al. 2006), laser-Doppler velocimetry (LDV) (Forte et al. 2006), laser-induced fluorescence (LIF) (Scime et al. 2005), and background-oriented schlieren (BOS) technique (Biganzoli et al. 2005).

As mentioned in the preceding paragraphs, flow separation and laminar transition to turbulence alter the value of the wall skin friction or shear stress. Blowing or suction as an effective boundary-layer control method, stabilizes the laminar boundarylayer and prevents separation, which accordingly reduces the wall shear stress. Hence, measurement of the wall shear stress is a means to evaluate the boundary-layer control effectiveness. Hot-film sensors have been used to measure the wall shear stresses. These sensors work based on the local determination of the instantaneous rate of heat transfer from a small source on the surface and the fluid (Armistead et al. 1968).

The plasma actuator's technology has been improved during the years, and it is predicted that they would be vastly implemented on the future wings or blades. At that time, automatic adjustment of the actuator parameters will become necessary. Hot-film sensors have some properties that make them most suitable for use with actuators to monitor the actuator control authority. These properties are, light weight, no surface disturbing due to being very thin, fast-response, on-field application capability (unlike optical methods), etc. None of the measurement methods mentioned in the preceding paragraph except Hot-film sensors, can be used as a built-in sensing method for a closed-loop boundarylayer control method by plasma actuators. Of course, there are some technical difficulties with using hot-film sensors close to the large electric field of the plasma actuators. Moreover, there is no 
vivid solution for the adverse effects of dust or other particles in the air on the performance degradation of the hot-film sensors.

This paper aims, for the first time, to discuss the results of experiments, which have been performed on the measurement of the wall shear stress downstream of a DBD plasma actuator in the steady actuation mode by several chord-wise located hotfilm sensors, in order to determine the boundarylayer control authority of the actuator on a windturbine blade section in deep-stall, post-stall and beyond-stall regions. This study governs the hotfilm data together with the surface pressure history to discover the underlying flow instabilities at high angles of attack. On the other hand, a DBD plasma actuator operating in its steady actuation mode is utilized to control the separated boundary-layer. Comparison of the controlled and uncontrolled results facilitates the determination of flow features on the wall. To the authors' knowledge, a similar analysis cannot be found in the literature.

The experiments were performed on a $2 \mathrm{D}$ windturbine blade-section model at a freestream velocity of $20 \mathrm{~m} / \mathrm{s}$ at different angles of attack from zero to beyond the deep stall region. Various states of the post-stall boundary-layer is identified using hot-film data, and the authority of the plasma actuator is evaluated in each case. A comprehensive discussion was made on the use of hot-film sensors on the same model and a novel transition detection method in publications by Akhlaghi et al. (2020). The results of this paper can be used to develop an on-line onboard measurement system to monitor the quasiwall shear stress on the surface of the blade and apply the effective actuation mode during the operation of the turbine.

\section{EXPERIMENTAL SETUP AND PROCEDURE}

All experiments were conducted in an open loop, low speed, and suction-type wind tunnel with a rectangular test section of $80 \times 100 \times 200 \mathrm{~cm}^{3}$. The flow speed in the test section varies continuously from 10 to $100 \mathrm{~m} / \mathrm{s}$. The present tests were conducted at a Reynolds number of $0.27 \times 10^{6}$. This Reynold number is selected because it is a common value in the nominal operating condition of most of the wind-turbines with the rated power of several hundred $\mathrm{kW}$. It should be noted that this tunnel is of an atmospheric type one and variations of Reynolds number cannot be accomplished through constant Mach number. The inlet of the tunnel has a $7: 1$ contraction ratio and is equipped with a honeycomb and a set of three monolith antiturbulence screens in its settling chamber to reduce the tunnel turbulence level to an acceptable value. The turbulence intensity of the incoming flow in the test section ranges from $0.1 \%$ to $0.3 \%$, depending on the freestream velocity. Temperature and total pressure of the freestream flow are $20{ }^{\circ} \mathrm{C}$ and 84 $\mathrm{kPa}$. The measurement inaccuracies of the freestream parameters are listed in Table 1.

Table 1 Freestream flow measurement Inaccuracies (\%).

\begin{tabular}{|c|c|c|c|c|}
\hline$\Delta T / T$ & $\Delta P / P$ & $\Delta \rho / \rho$ & $\Delta U_{\infty} / U_{\infty}$ & $\Delta R e / R e$ \\
\hline 0.033 & 1.421 & 0.035 & 2.009 & 2.010 \\
\hline
\end{tabular}

A critical section of a $660 \mathrm{~kW}$ wind-turbine blade with $25 \mathrm{~cm}$ chord and $80 \mathrm{~cm}$ span has been used in this investigation, as shown in Fig. 1. The model is constructed of fiberglass with a measured accuracy of $\pm 0.1 \mathrm{~mm}$. To measure the pressure distribution over the surface of the model, 63 pressure taps were used, congested at the leading-edge (Akhlaghi et al. 2020). Meanwhile, for measurement of the time history of wake and velocity behind the model, a rake is installed at $1.5 \mathrm{c}$ downstream of the model. The rake data is also used for measurement of the drag of the model. The surface and rake pressures have been measured using differential pressure transducers with ranges from 0.075 to $1.0 \mathrm{psi}$ and with a maximum uncertainty of $0.15 \%$ of their full span.

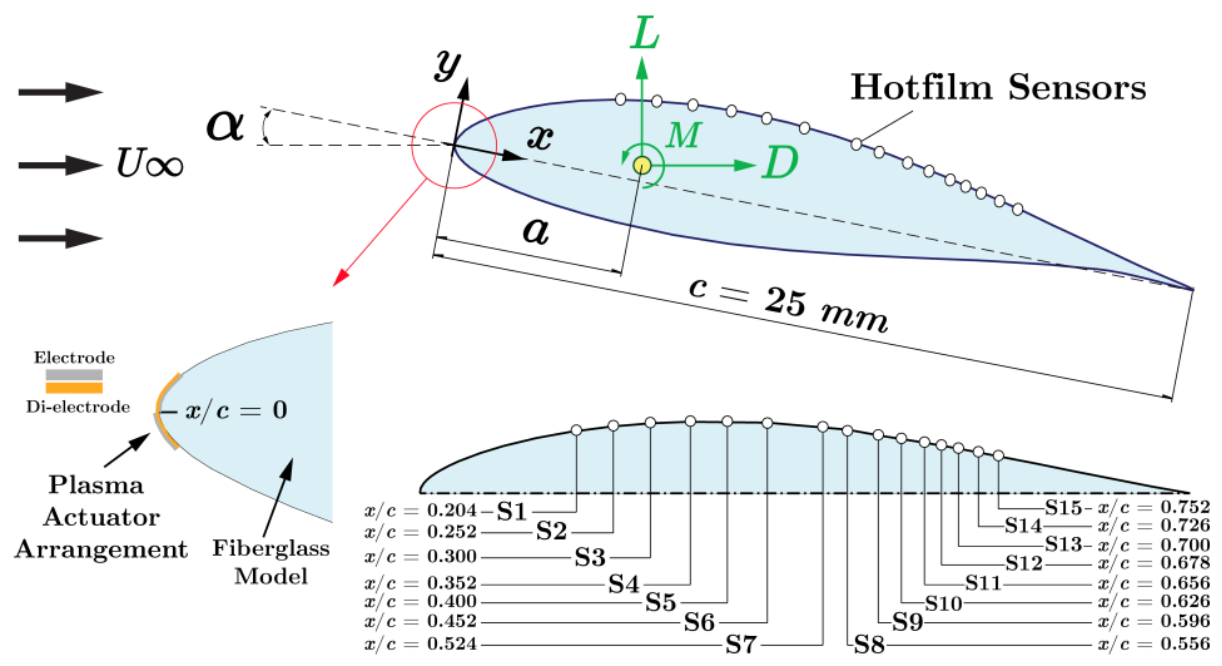

Fig. 1. Schematic of model together with hot-film sensors and plasma actuator arrangement and location. 
Pressures are sampled at a rate of one $\mathrm{kHz}$. Fifteen hot-film sensors were glued on the airfoil's upper surface at locations of $0.204 \leq x / c \leq 0.752$. The arrangement of the hot-film sensors together with their position are illustrated in Fig. 1. The model was installed vertically inside the test section on a wind-turbine blade motion simulator designed, built, and calibrated for these tests. The system is capable of producing a wide range of complicated dynamic motions as well as the static angles of attack variations. Figure 2 shows the integration arrangement of the blade motion simulator with the wind-tunnel test section and the model. An electrical panel is attached to the main frame of the simulator, including motor drivers, PLC, terminals, switches, indicators, etc. (Fig. 2a). Figures $2 b$ and c show the arrangement of the rake assembly and detailed view of the rakes. Static and total rakes are installed $2.5 \mathrm{c}$ behind the model. An angle of attack measurement unit is installed on the ceiling of the test section which is shown in Fig. 2d. In this study, no dynamic motion is exerted on the model, and the tests are fully static, pitch-pause motion. The angle of attack of the model was measured using a 14-bit digital encoder with an accuracy of $0.022^{\circ}$. The accuracy of the angle of attack regulation is $0.0013^{\circ}$.

To measure the pressure distribution over the surface of the model, 63 pressure-taps were used, congested at the leading edge. The position of the transducers and pressure tap configurations are discussed in detail in (Mahdavi et al. 2020 and
Akhlaghi et al. 2020). The surface pressures have been measured using differential pressure transducers with ranges from 0.075 to $1.0 \mathrm{psi}$ and maximum uncertainty of $0.15 \%$ of full span. The analog voltage output of the transducers was transferred to an A/D board, converted to digital signals, and saved to the data-acquisition computer.

Hot-film sensor arrangement on the model is shown in the Fig. 2d. The un-calibrated hot-film data were used to define the quasi-wall shear stress and provide semi-quantitative information about the state of the boundary-layer. All hot-film sensors were sampled at a frequency of $5 \mathrm{kHz}$. High values of overheat ratio may lead to the film deflection that would affect both the shear layer and the neighboring sensors. On the other hand, very low over-heat-ratio values would result in the low sensitivity of hot-film sensors to the flow disturbances. Based on the literature survey, manufacturer recommendation, and previous experiences, the overheat ratio in these tests is set to 0.9 . Every raw data was digitally filtered by a lowpass filtering routine to eliminate white-noise effects from the signal. During the filtering process, the cut-off frequency was obtained using power spectrum estimation or the frequency-domain analysis. The white noise leads to a constant power spectral density. All data from the encoder, pressure transducers and hot-film sensors were recorded simultaneously via an A/D board (Akhlaghi et al. 2020).

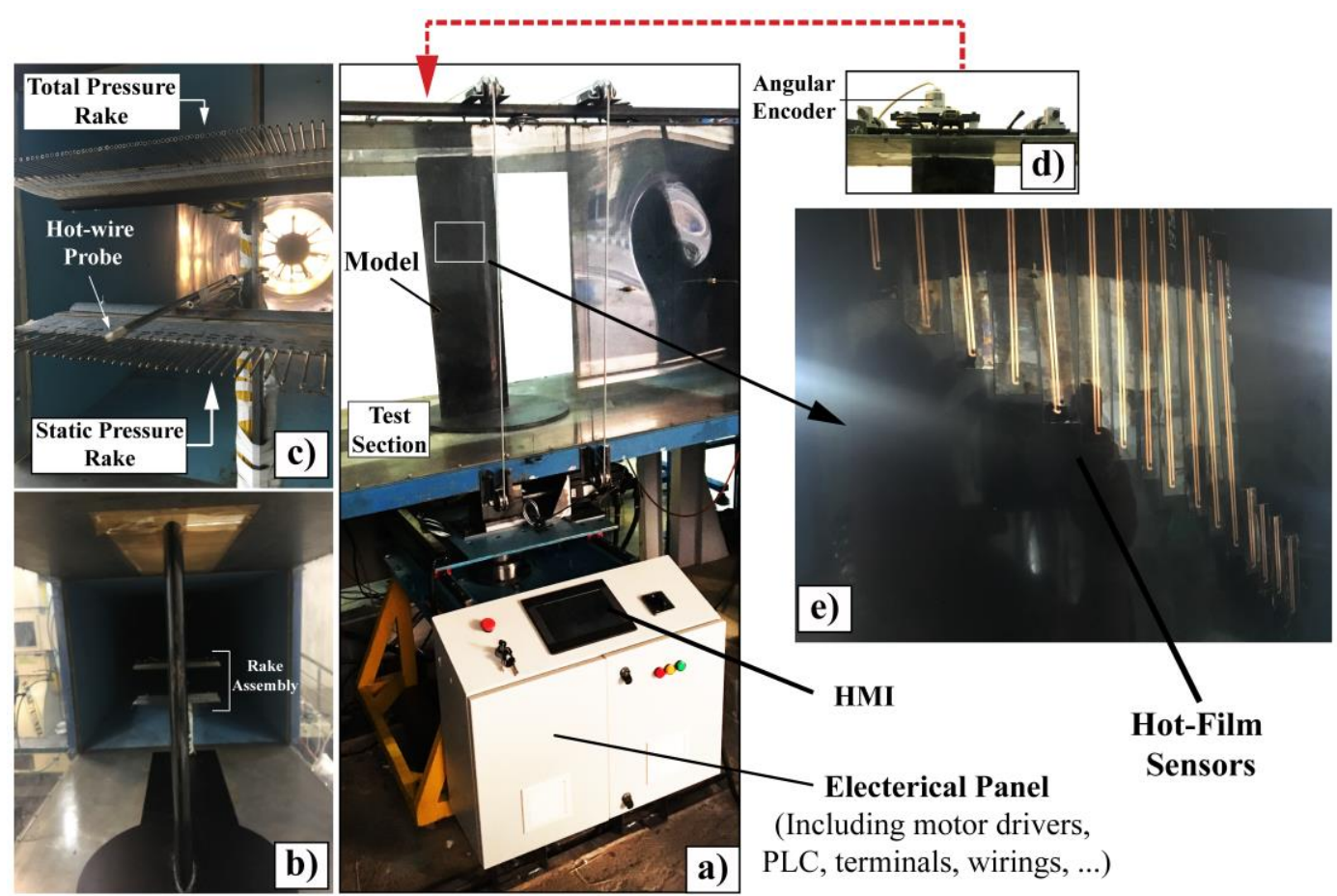

Fig. 2. a) Installation Arrangement of wind-turbine blade model on the motion simulator and in the test section, b) Model and rake assembly arrangement c) Rakes and hot-wire probe arrangement, d) Angle of attack measurement unit on the ceiling of test section e) Arrangement of hot-film sensors. 


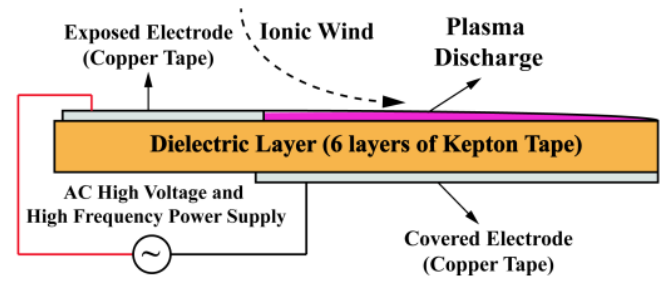

Fig. 3. Schematic of DBD plasma actuator arrangement.

To drive the DBD plasma actuator, a lab-made HV power supply was used. The high-voltage (HV) source is adjustable for different waveforms, frequencies ( 0 to $50 \mathrm{kHz}$ ) and voltages (0 to 25 $\mathrm{kV}_{\mathrm{pp}}$ ). The peak-to-peak AC voltage to the plasma actuator was set to $16 \mathrm{kV}_{\mathrm{pp}}$ in the current experiments. The input waveform was of sinusoidal type with an AC frequency of $2.17 \mathrm{kHz}$. The actuator consisted of two 0.0254-mm-thick coppertape layers separated by 6 layers of $0.127 \mathrm{~mm}$-thick layer of Kapton tape. The schematic of the DBD plasma actuator used in this experimental study is shown in Fig. 3. The arrangement and location of the actuator on the model are illustrated in Fig.1. The applied voltage to the electrodes was measured by an HV probe. The voltage signals were recorded by a four-channel digital oscilloscope. Further details on the plasma source used in the present study can be found in (Mahdavi et al. 2020).

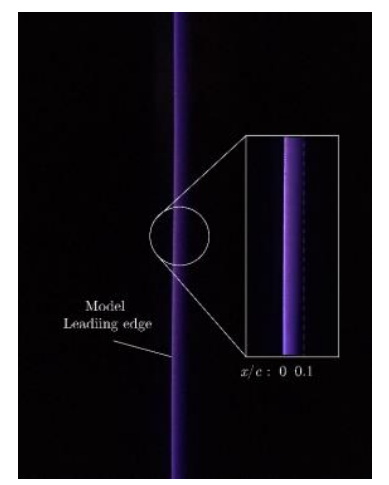

Fig. 4. Plasma discharge on the model surface $\left(\mathrm{V}_{\mathrm{pp}}=16 \mathrm{kV}, f_{\mathrm{ac}}=2.19 \mathrm{kHz}\right)$.

All experiments were conducted at a constant Reynolds number of $0.27 \times 10^{6}$, which corresponds to the freestream velocity of $20 \mathrm{~m} / \mathrm{s}$. Surface pressure measurements along with the rake total and static pressure as well as the hot-film measurements were performed for the case where the plasma actuator was absent, i.e. clean model. This case is called the baseline configuration. During this phase, all sensors and data-acquisition hardware were checked, and the experimental results were verified. The repeatability of the measurements were also examined. In the next step, the plasma actuator was added to the airfoil leading edge $(x / c=$ $0 \%$ ) with $10 \%$ overlap. The measurements were performed for the cases of plasma-ON and plasmaOFF for a range of angle of attacks of $0 \leq \alpha \leq 35^{\circ}$. The AC carrier frequency $\left(f_{\text {ac }}\right)$, peak-to-peak voltage $\mathrm{V}_{\mathrm{pp}}$ and the location of the actuator is selected experimentally.

The average velocity of the electric wind was measured by using a pitot tube. Since a commercial pitot tube is made of stainless steel, it may lead to spark at distances near the discharge surface, so a glass capillary tube with the inner diameter of 0.5 $\mathrm{mm}$ was used to measure the total pressure. A $2 \mathrm{D} \mathrm{x}-$ $\mathrm{z}$ positioner with 0:01 $\mathrm{mm}$ accuracy was employed to displace the glass tube along the horizontal and vertical axes. Before positioning the actuator on the model surface, a large number of experiments were made to measure the induced velocity, for different voltage-current shapes, peak-to-peak voltages and AC carrier frequencies. Greatest induced velocities and longest extension were reached in the highest peak-to-peak voltage achievable by the source. The same experiments were also made on the AC carrier frequency. Frequencies of $2.17 \mathrm{kHz}$ and $5 \mathrm{kHz}$ were tested on the model, and the best aerodynamic performance was achieved, in $f_{\mathrm{ac}}=2.17 \mathrm{kHz}$. More details on the performance of the power-source and actuator in both steady and pulsed actuation modes can be found in (Mahdavi et al. 2020).

The best position of the plasma actuator is also examined. In the preliminary stages of the tests, plasma actuators were located in the positions $x / c=$ $0.3, x / c=0.4$ and $x / c=0.6$. These positions were selected to examine whether the actuator can affect the flow separation or transition when it located near the separation point in the angles of attacks near stall or not. Actuator's performance in these positions was disappointing. Finally, the plasma actuator was located in $x / c=0$, and an appropriate control authority were found.

Figure 4 shows the image of the plasma discharge on the model surface and the extension of the plasma taken in a dark lab. The smooth discharge of the plasma along the model span guarantees the appropriate selection and installation of the electrodes on the surface.

\section{EXPERIMENTAL RESULTS \& DISCUSSIONS}

\subsection{Surface pressure measurement and wake survey}

The lift coefficient is calculated by integration of the measured surface pressures, 64 pressure ports, along the airfoil chord. For further description of the lift calculation, using surface pressure integration refer to (Anderson et al. 1991). The wake survey is a common technique for measuring the profile drag of a 2-D model. The wake survey measures static pressures and the total pressure within the wake and compares those values to the freestream total pressure. The total pressure deficit, or essentially the momentum loss of the flow, can then be directly related to the profile drag. The wake pressure deficit has to be measured sufficiently far from the trailing edge of the model to allow the static pressure to recover to freestream conditions. Further corrections can be applied to adjust for the static pressure in the wake not being 
fully recovered at the location of the measured wake survey (Schlichting et al. 1960).

Figure 5 compares the measured lift and drag coefficients of the airfoil for both plasma-ON and plasma-OFF cases. As is shown in Fig. 5a (plasmaOFF), three main regions in the $C_{l-\alpha}$ can be identified. First region is the linear part of the $C_{l-} \alpha$ plot. In this region, the lift coefficient linearly increases with the angle of attack. This region ends about an angle of attack of $10^{\circ}$. The other region is called light stall region where the lift coefficient remains nearly constant as the angle of attack is increased. In this region, the flow separation point over the airfoil surface remains in the range of $x / c=$ $0.4-0.5$. Flow in this region is somehow unstable and is sensitive to the freestream turbulence and surface roughness. As the angle of attack is further increased, a range of unfavorable-pressure-gradient on the airfoil surface $(d P / d x>0)$ due to the flow separation appears over the surface of the model. For an angle of attack of about $20^{\circ}$, the flow

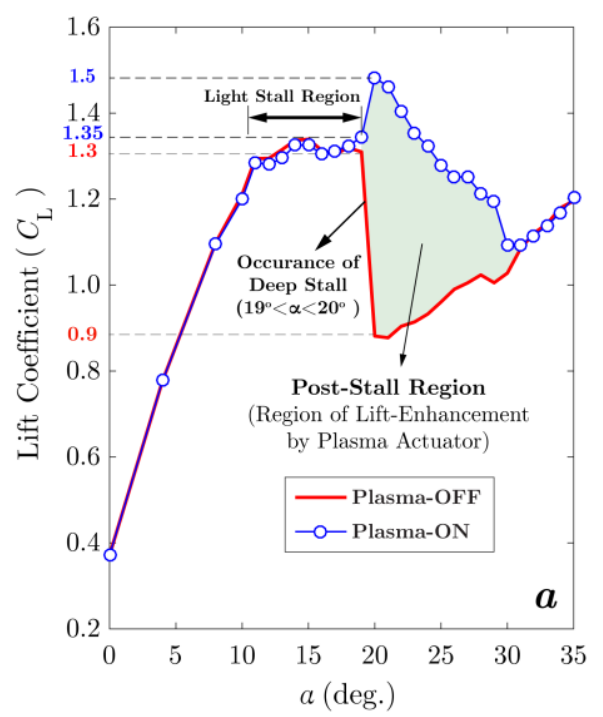

momentum can't overcome the existing adversepressure-gradient and the flow separation point suddenly moves towards the leading edge. This angle of attack is the onset of occurrence of the deep stall phenomenon.

Figure 6 shows the pressure coefficients on the blade section surface for the deep stall angle of attack $\left(\alpha=19^{\circ}\right)$, beyond the stall angle of attack of this airfoil. This figure, confirms the aforementioned descriptions. When the plasma actuator is off, at $\alpha=19^{\circ}$ the separation point is somewhere about $x / c=0.4$. However, for $\alpha=20^{\circ}$, the separation suddenly moves upstream towards the leading-edge $(x / c=0)$. The region of the $C_{l}-\alpha$ plot, next to this point is called, post-stall region, Fig. 5a. Figure 6 shows that for the plasma-OFF case, in the post-stall region, the separation point remains near the leading-edge. Vast flow separation in the stalled region leads to the sudden reduction of the lift coefficient to about $67 \%$ of the lift coefficient for $\alpha=19^{\circ}$.

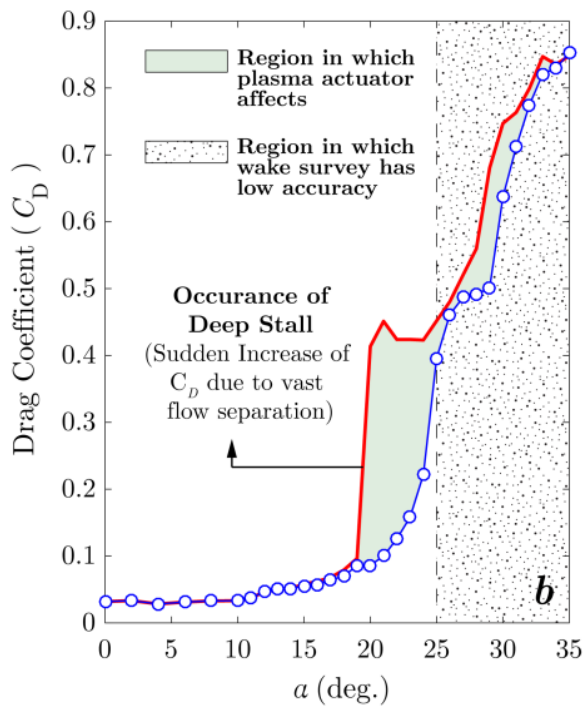

Fig. 5. Comparison of a) lift and b) drag coefficients in plasma-ON and plasma-OFF cases.
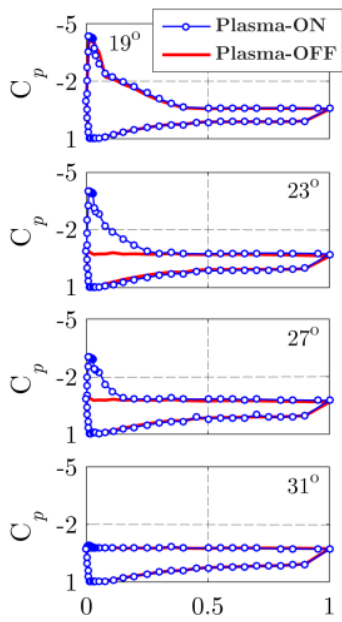

$x / c$
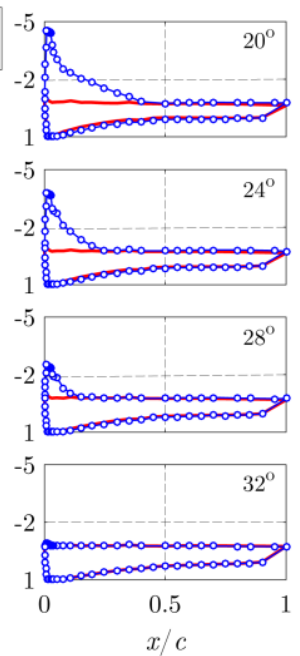
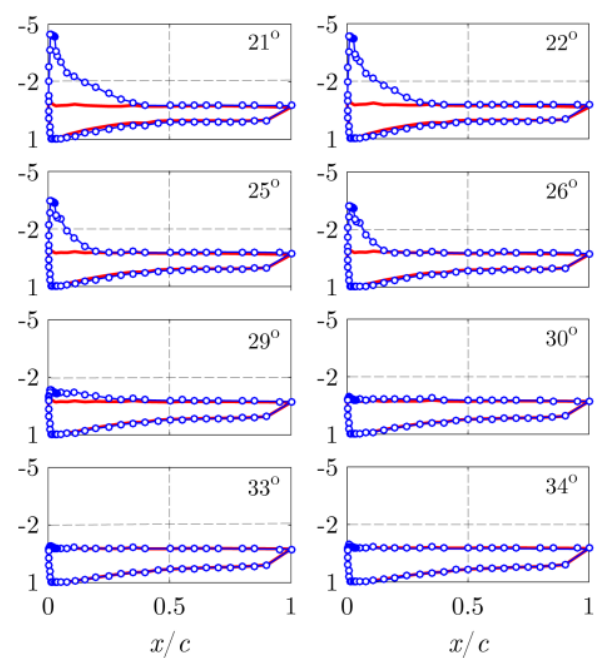

Fig. 6. Pressure coefficient distribution on the blade section surface in deep stall and post-stall angles of attack for plasma-ON and plasma-OFF cases. 
Figure $5 b$, shows the blade-section drag-coefficient versus angle of attack. This drag coefficient is calculated using the wake survey method. Drag coefficient gently increases with angle of attack, while, in the deep-stall point $C_{d}$ is seem to improve drastically $\left(\alpha=19^{\circ}-20^{\circ}\right)$. The flow separation increases the static pressure on the upper surface and subsequently increases the pressure drag as seen from Fig. 5 and Fig. 6. Because static pressure recovery in the test section cannot be obtained in the location of pressure rake, wake survey method has less accuracy for angles of attack greater than $25^{\circ}$. This region is specified by black dots in Fig. $5 b$.

Figures 5 and 6 show that plasma actuator can effectively control the flow separation and enhance the aerodynamic force coefficients in the post-stall region. The plasma actuator used in this study is a steady plasma actuator. In the steady plasma actuation mode, the basic control mechanism is through the momentum insertion (Abdollahzadeh et al. 2018).

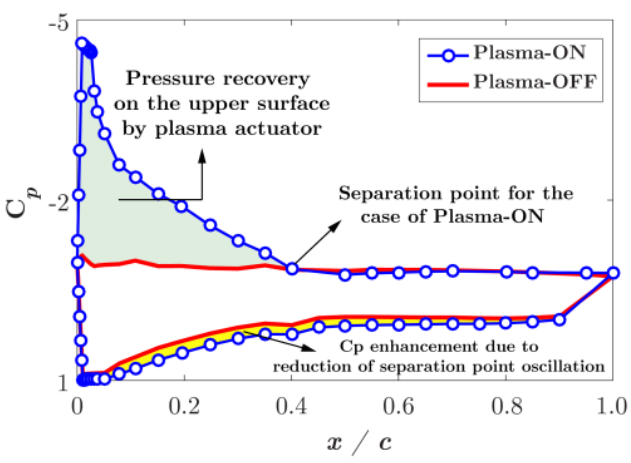

Fig. 7. Comparison of pressure coefficient distribution of Plasma-ON and Plasma-OFF cases for $\alpha=20^{\circ}$.

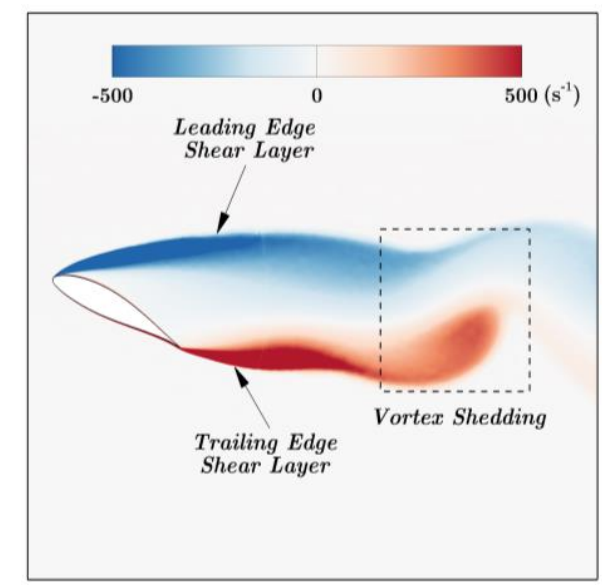

Fig. 8. Contour of $Z$-vorticity for a post-stall angle of attack derived from numerical simulation: illustration of leading-edge and Trailing-edge shear layer formation and vortex shedding.

As previously mentioned, by increasing the angle of attack from $\alpha=19^{\circ}$ to $\alpha=20^{\circ}$, the flow separation point jumps towards the leading-edge. However, for the plasma-ON situation, the momentum is inserted into the boundary-layer and the flow kinetic energy near the surface increases. This kinetic energy neutralizes the unfavorable-pressure-gradient. As a result, the flow reattaches and the separation point are pushed downstream toward $\mathrm{x} / c=0.4$. The pressure distribution shown in Fig. 6 for $\alpha=20^{\circ}$ clarifies the above statement. A closer look at this effect is shown in Fig. 7.

Figure 5a depicts that the plasma actuator for the deep-stall angle of attack not only prevents the lift reduction, but also it increases the lift coefficient to some extent (above $10 \%$ of the lift coefficient at $\alpha=$ $19^{\circ}$ for the plasma-ON case). It is expected that the plasma actuator energizes the boundary-layer and pushes the separation point downstream nearly to the location where it was for the $\alpha=19^{\circ}$ case and at its limit the lift coefficient for $\alpha=20^{\circ}$ becomes equal to the lift coefficient at an angle of attack of $\alpha$ $=19^{\circ}$. Figure 7 compares the pressure distribution on the airfoil for the cases of Plasma-ON and Plasma-OFF for $\alpha=20^{\circ}$. It shows that for the Plasma-ON case pressure on the lower surface of the airfoil is slightly increased too. This lowersurface pressure enhancement increases the lift coefficient value. Similar results were also reported in Post et al. (2004) and Little et al. (2018) but no discussion about the cause of this observation is given in these references. It may be related to the specific dominating mechanisms associated with the post-stall flow. It has been verified that two major mechanisms play the essential role in the fully separated flow field: the local instability of the separated shear layers from both leading and trailing edges, and the global instability that causes vortex shedding (Wu et al. 1998). These flow phenomenon are depicted in Fig. 8, which shows the $\mathrm{z}$ component of the vorticity $\left(\omega_{\mathrm{z}}\right)$ around the airfoil in a post-stall angle of attack derived from CFD simulations. The sudden jump of the separation point from $x / c \approx 0.4$ (pre-stall separation point) to the leading edge with an increase of the angle of attack in the post-stall region (Fig. 6) leads to the roll-up of the shear layer at both leading and trailing edges, hence the vortex shedding phenomena would occur. It is hypothesized that the presence of the shear layer and vortex shedding instabilities together with the occurrence of secondary and tertiary separations may lead to the excitation of the laminar sub-layer on the pressure side of the airfoil which eventually leads to the creation of the extended laminar separation bubbles. Then flow instabilities convect from the leading and the trailing edges of the airfoil through the lower surface boundary-layer. The plasma actuator eliminates the underlying mechanisms of the poststall separated flow and subsequently recovers the pressure on the lower-surface of the airfoil.

\subsection{Hot-film Measurement}

Figure 9 a-f shows the pressure distribution on the suction side of the airfoil together with the root mean square (RMS) value from the voltage output of the hot-film sensors at deep-stall and at various post-stall angles of attack for both plasma-ON and 
plasma-OFF cases. The RMS of a signal is a suitable means of cumulative oscillation amplitude measurement. This parameter describes the strength of an alternating signal (Soltani et al. 2016). In the deep-stall angle of attack $\left(\alpha=19^{\circ}\right)$, Vrms is nearly constant across the chord and its value is very small. Flow in this case is not affected by any significant instabilities. The pressure distribution of Fig. 9a shows that separation in this case occurs about $x / c \approx 0.4$. No separation point fluctuation is noticed in this case. In this case, $\alpha=19^{\circ}$, the plasma actuator is seen to have no effects on the flow structure. It was mentioned previously that the current plasma actuator is operated in the steadyactuation mode, and the imparting momentum to the boundary-layer is the main control mechanism in this DBD plasma actuator. As the separation point is located about $8 \mathrm{~cm}$ downstream of the actuator (which is located on the leading-edge), the applied momentum cannot energize the separated boundary-layer and moves it downstream.
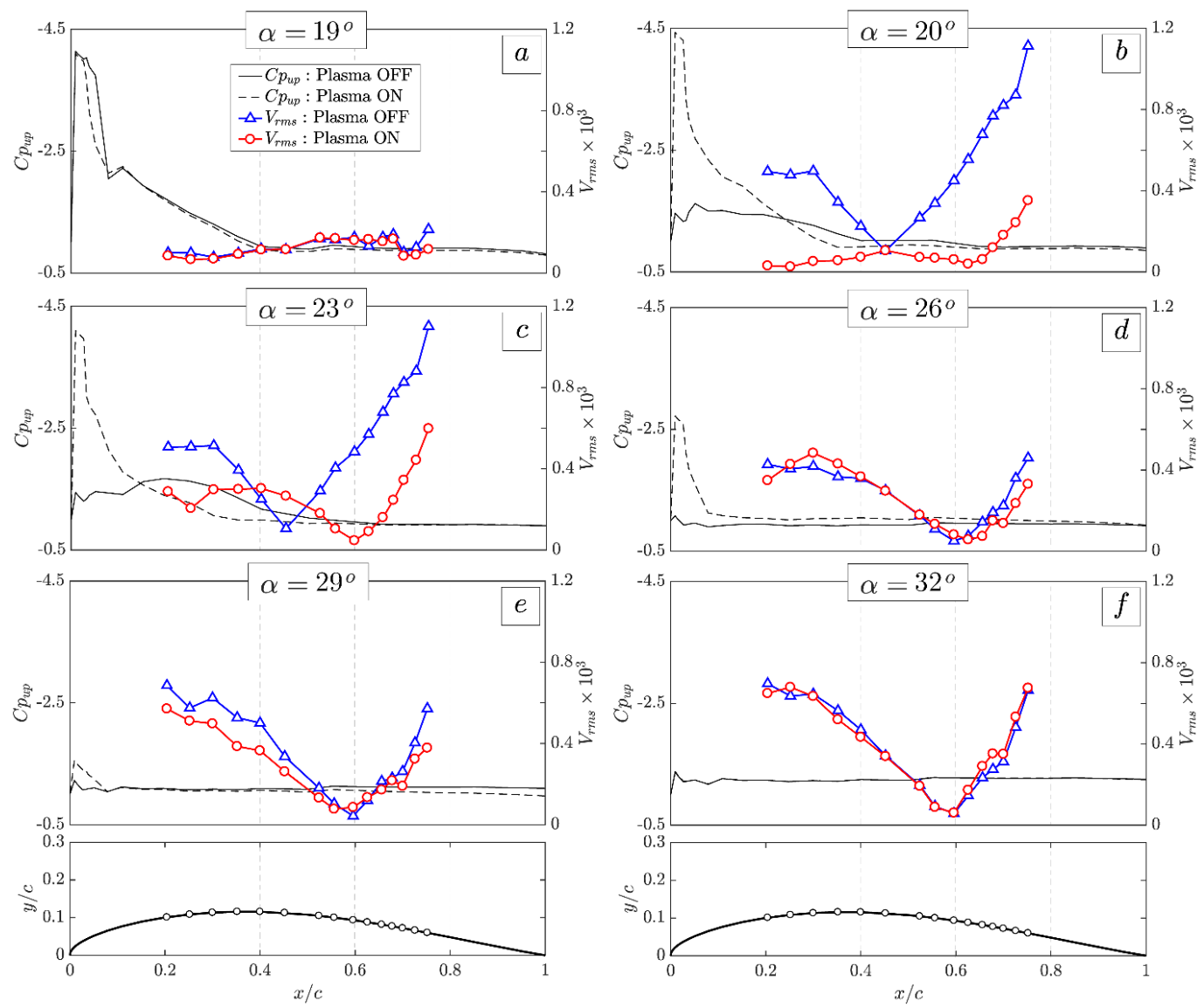

Fig. 9. Suction-side pressure coefficient distribution together with RMS value of hot-film sensor voltage. $\left(\mathrm{V}_{\mathrm{pp}}=16 \mathrm{kV}, f_{\mathrm{ac}}=2.19 \mathrm{kHz}\right)$.

As the angle of attack is increased to $\alpha=20^{\circ}$, the separation point moves upstream towards the leading-edge and as the result, the unfavorablepressure-gradient becomes stronger and detaches the flow further; $x / c \approx 0.4$ is the location of maximum thickness of the present airfoil. When the separation point moves upstream of this point, maximum thickness, it cannot stay stable between the leading-edge and this point, $x / c \approx 0.4$, and inevitably the separated shear layer moves towards the leading edge, and the flow became fully separated. Consequently, the magnitude of the pressure on the suction side of the airfoil, hence the lift force drops significantly (Fig. 9b and Fig. 5a). Although, the surface pressure on the suction side in the case of plasma-OFF for this angle of attack decreases considerably, but the values of pressurecoefficients between the leading-edge, and the location of the maximum thickness is still less than
-1 which, may indicate that the separation point fluctuates in this region. The separated shear layer fluctuates and as the Kelvin's condition necessitates, the trailing edge shear layer fluctuates too and time variations of the circulation on the airfoil may lead to the shedding of the vortices behind the model. As Fig. 9b shows, the hot-film sensors sense the shear layer fluctuations. The sensors closer to the leading and trailing edge are affected more while those located near the maximum thickness are less affected. The points upstream of $x / c \approx 0.4$ are affected by the leadingedge flow instabilities, and the points downstream of this location are affected by the trailing-edge flow instabilities. As a result, the shear layer instability in this point is neutralized. The application of the plasma actuator, forces the separation point to move to its pre-stall position and eliminates the flow instabilities. The hot-film 
sensors effectively capture this effect. As Fig.9b indicates, the plasma actuator is seen to eliminate the leading-edge instabilities completely, and it further reduces the flow instabilities near the trailing edge, however, the hot-film sensors still show some oscillations. It is believed that the trailing-edge shear layer instabilities are coupled with the leading-edge shear layer instabilities and the increase of geometric angle of attack increases the differences between the local flow speeds around the shear layer. Therefore, the trailing-edge shear layer rolls up and leads to some weak instabilities (increase of $\mathrm{V}_{r m s}$ near the trailing-edge as seen from Fig. 9b).

As the angle of attack is further increased, the unfavorable-pressure-gradient increases while the control authority of the plasma actuator decreases. For $\alpha=23^{\circ}$ the plasma actuator is able to move the separation point downstream and eliminates the corresponding instabilities, however, the RMS level of the hot-film signals increases in comparison with the values for the case of $\alpha=20^{\circ}$. It seems that due to the effects of the reverse flow near the surface which is relatively strong for this angle of attack, $\alpha$ $=23^{\circ}$, the separation point around the point of maximum thickness fluctuates and a self-exciting flow alteration takes place. The plasma actuator moves the separation point downstream by momentum insertion while the presence of the reverse flow near the airfoil surface pushes it upstream. This loop continues to replicate, and the separation point fluctuates back and forth.

With the further increase of the angle of attack, the control authority of the plasma actuator decreases and the separation point stabilizes at the leading edge. The separation shear layer empowers as the angle of attack is increased, however, the hot-film sensors show a lower amount of instability in comparison with those at lower angles of attack for both plasma-ON and plasma-OFF cases. In these cases, it is hypothesized that the flow separates exactly from the leading-edge, and the shear layer instabilities convect downstream. However, the upper surface of the airfoil is not directly affected. Of course, the instabilities spread in the fluid

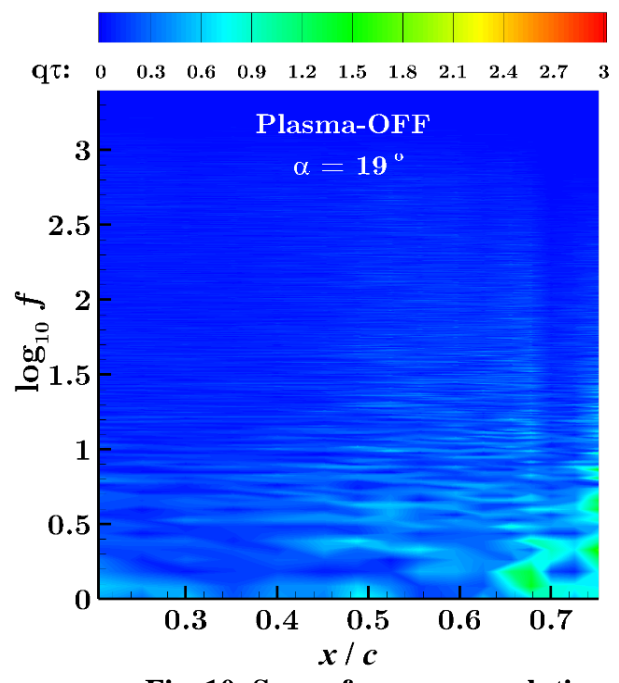

medium, however, the surface hot-films are affected much less as they did for the cases of lower angles of attack. The effects of both leading-edge and trailing-edge instabilities are seemed to be neutralized around $x / c \approx 0.6$ for higher angles of attack.

Contours of space-frequency evolution of the zeromean quasi-wall shear stress $(q \tau)$ on the suction side of the airfoil are used to identify the location of occurrence of various phenomenon along with their frequencies. The quasi-wall shear stress is derived from the uncalibrated hot-film data and is defined as below.

$q_{\tau}=\left(\frac{E^{2}-E_{0}{ }^{2}}{E_{0}{ }^{2}}\right)^{3}$

Where $E$ is the hot-film output voltage, and $E_{0}$ is the voltage for zero-flow condition or wind-tunnel-off case. Quasi-wall shear stress is a parameter derived from voltages of the hot-film sensor. However, the value is not exactly equal to wall shear stress. This parameter is defined to show a representation of wall shear stress using hot-film sensor measurement. Calibration of hot-film sensors is not that easy using a straight-forward method. So quasiwall shear stress is a parameter which is proportional to wall shear stress. This variable is used for a semi-quantitative study of the states of the boundary-layer. Further details and discussion of this equation and its application can be found in Akhlaghi et al. (2020).

Figure 10 demonstrates variations of $q \tau$ for deepstall angle of attack $\left(\alpha=19^{\circ}\right)$. It should be noted that the mean value of the signal shown in this figure is zero (mean value is subtracted). As it was previously discussed, in this case no significant instabilities can be found. Meanwhile, the hot-film sensors don't show any oscillations around the point $x / c \approx 0.4$ which confirms that the separation point is relatively stable. Some weak flow intermittencies is found near the trailing-edge which might be due to the inherent instabilities of the trailing-edge shearlayer.

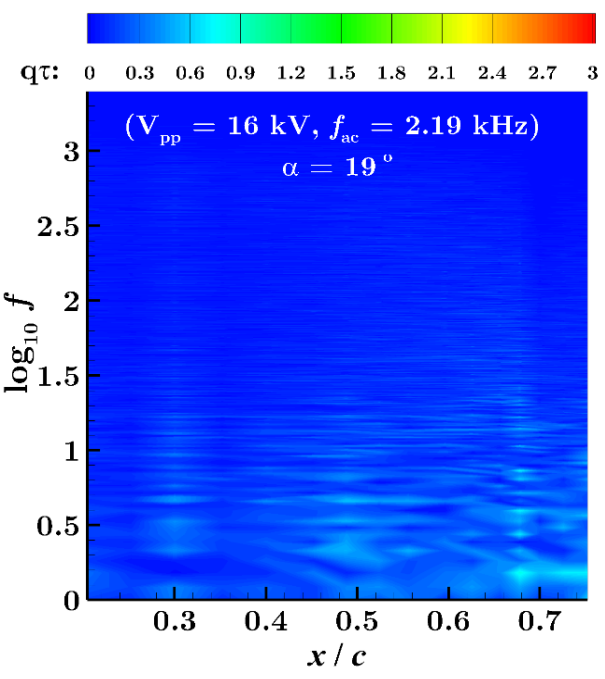

Fig. 10. Space-frequency evolution of quasi-wall shear stress signal: $\alpha=19^{\circ}$. 

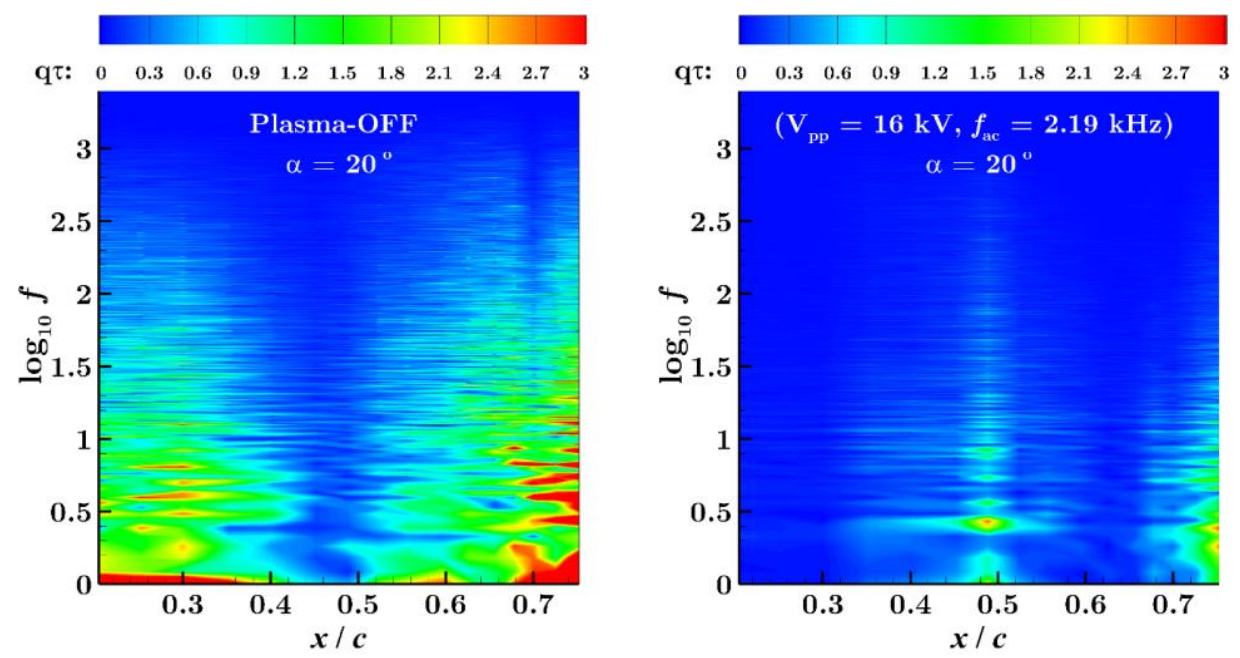

Fig. 11. Space-frequency evolution of quasi-wall shear stress signal: $\alpha=20^{\circ}$.
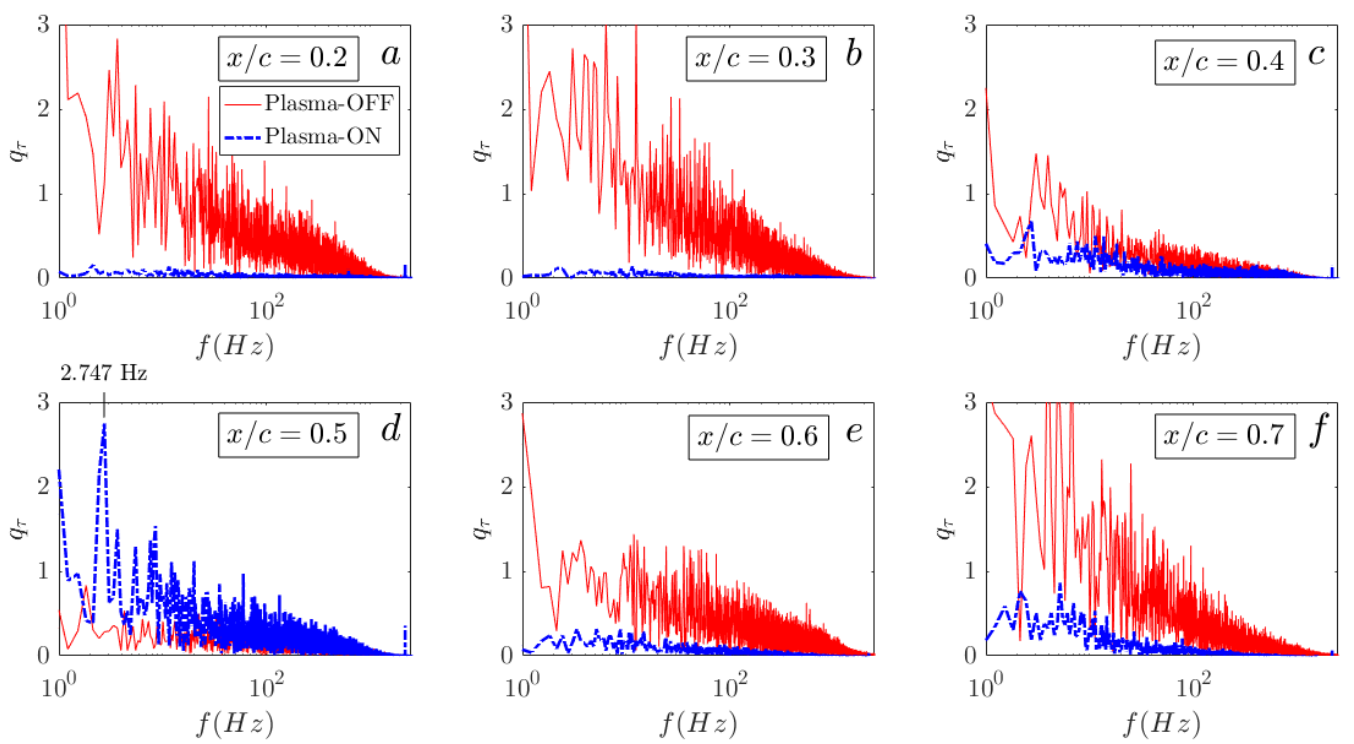

Fig. 12. Frequency Spectra of quasi-wall shear stress signal: $\alpha=20^{\circ}$.

As the deep stall phenomenon occurs $\left(\alpha=20^{\circ}\right)$, Fig. 11 , some instabilities with higher amplitudes take place near both the leading and the trailing edge of the airfoil. Near the leading-edge, the instabilities have low frequencies, mainly about $1 \mathrm{~Hz}$. A range of frequencies with moderate amplitudes are active in $x / c=0-0.4$. Figure 12 compares the frequency spectra of $q_{\tau}$ at different locations for both plasma$\mathrm{ON}$ and plasma-OFF cases. This figure shows that near the leading-edge $(x / c=0.2$ and $x / c=0.3)$ low frequencies around $1 \mathrm{~Hz}$ has greater amplitude. Moving towards the trailing-edge, these low frequencies $(f=1-10 \mathrm{~Hz})$ are eliminated and moderate frequency oscillations $(f=10-100 \mathrm{~Hz})$ appear near the trailing-edge. The low-frequency oscillations near the leading-edge are due to the reciprocation of the separation point. The same frequencies are active near the trailing-edge, but their amplitudes are higher. No significant oscillations are seen downstream of the separation point $(x / c=0.4$ and $x / c=0.5)$. As Fig. 11 demonstrates, the plasma actuator exhibits appropriate control authority in this case and pushes the separation point beyond the maximum thickness point and eliminates both the leading-edge and the trailing-edge instabilities. A low-frequency instability is seen in the case of plasma-ON near the separation point which might be due to the low amplitude reciprocation of the separation point. A frequency of $2.747 \mathrm{~Hz}$ is seen in Fig. 12d at a location of $x / c=0.5$ which is the separation-point location for the plasma-ON case. An important point is that the separated flow is a highly nonlinear multi-frequency system, and no distinct frequency can be reported for this case. This phenomenon is due to the coexistence of the shear layer frequency and the natural shedding frequency between the leading-edge shear layer and the trailing-edge vortices, plus the fluctuating frequencies of the secondary and tertiary separations (Wu et al. 1998). 

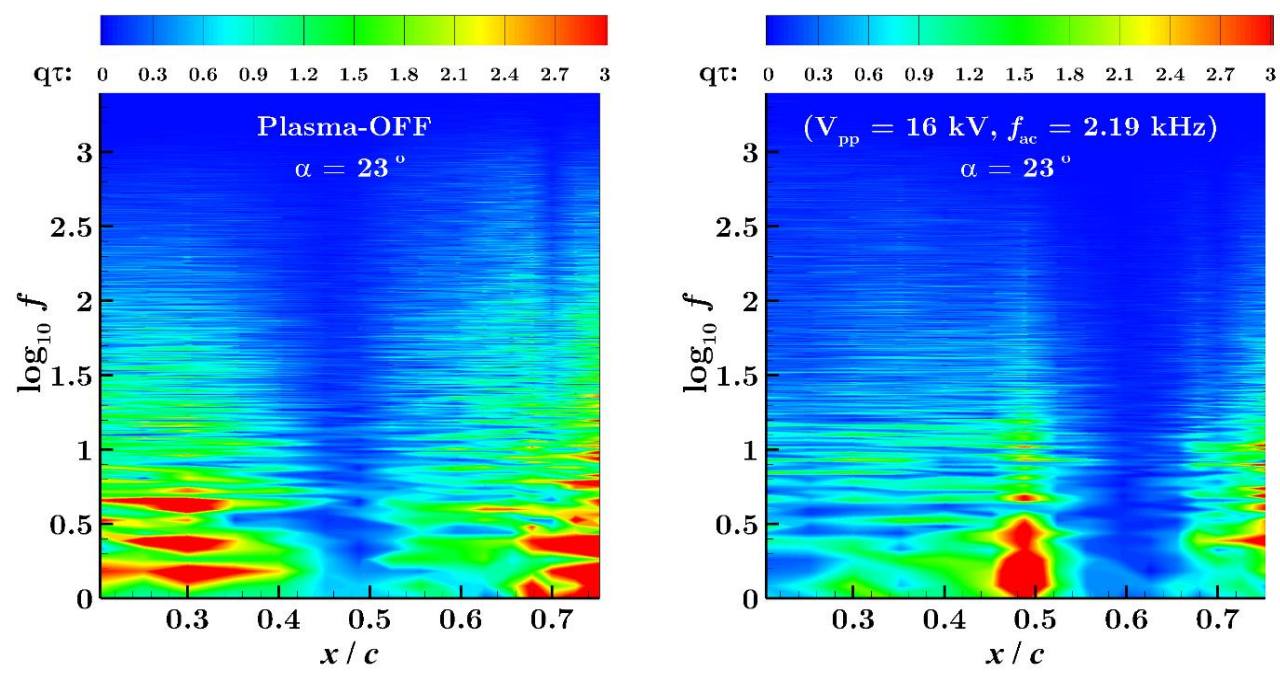

Fig. 13. Space-frequency evolution of quasi-wall shear stress signal: $\alpha=23^{\circ}$.

Insomuch as the interactions of these frequencies are not mutually integer multiples of each other, which implies chaos, the post-stall separated flow often exhibits a random behavior. Figure 13 demonstrates that as the angle of attack is further increased to $\alpha=23^{\circ}$, the flow near the leading-edge becomes more chaotic and several frequencies with considerable amplitudes are revealed. The spectra of Fig. 14 illustrates that the hot-film sensors show some active frequencies in the range of $1-5 \mathrm{~Hz}$. Figure 14 compares the spectra of hot-film signals for the cases of plasma-ON and plasma-OFF at an angle of attack of $23^{\circ}$ and at $x / c=0.3$.

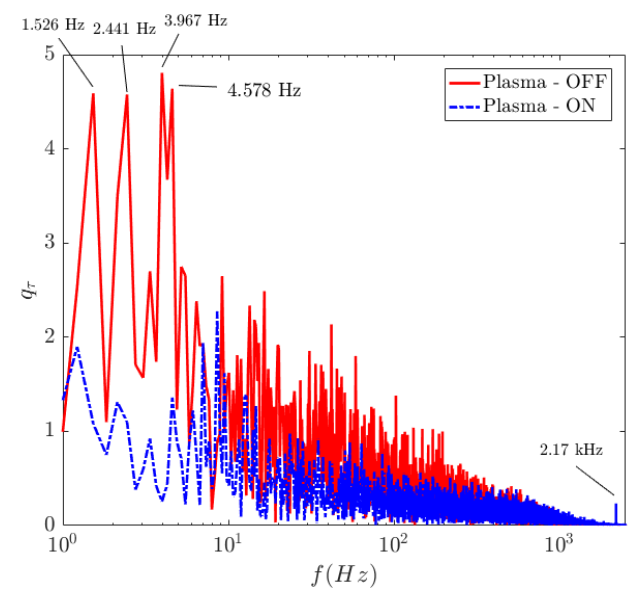

Fig. 14. Frequency spectra of quasi-wall shear stress, $\alpha=23^{\circ}$ and $x / c=0.3$.

Wu et al. (1998) reported low-frequency periodic oscillations near the onset of the static stall. As it is concluded in their study along with the present hotfilm data, the origin of the flow fluctuations traces to the upper surface of the airfoil and is associated with a periodic switching between the stalled and the un-stalled states. It was mentioned in the previous descriptions of the RMS values that the separation point reciprocates between the leadingedge and the maximum thickness of the airfoil, $x / c$ $\approx 0.4$ for the present airfoil. The experimental results of Zaman et al. 1989 had rejected the possibility of interference of fan instabilities, acoustic standing waves or structural resonances in the occurrence of these low-frequency oscillations (Zaman et al. 1989). The separation control authority of the plasma actuator for the case of $\alpha=$ $23^{\circ}$ is reduced to some extent. It means that although the actuation stabilized the flow, but some frequencies with moderate amplitudes are observed near the leading-edge, Fig. 14. Moreover, the amplitude of the reciprocation of the separation point on the surface increases, to some extent, for the plasma-ON case too. The low-frequency flow instabilities can also be found, but the location of their occurrence is moved upstream. The range of active frequencies in this condition is nearly equal to those of the plasma-OFF case. The lowfrequency phenomena move to the point of separation. Therefore, it could be concluded that the source of this oscillation is the reciprocation of the separation point due to a self-exciting mechanism on the upper surface of the airfoil. Of course, the free-stream turbulence has an essential rule in the excitation of such instabilities. At the onset of the post-stall, the receptivity of the boundary-layer increases and the freestream turbulence penetrate the sublayers and as a result; the so-called selfexciting flow instabilities are exited.

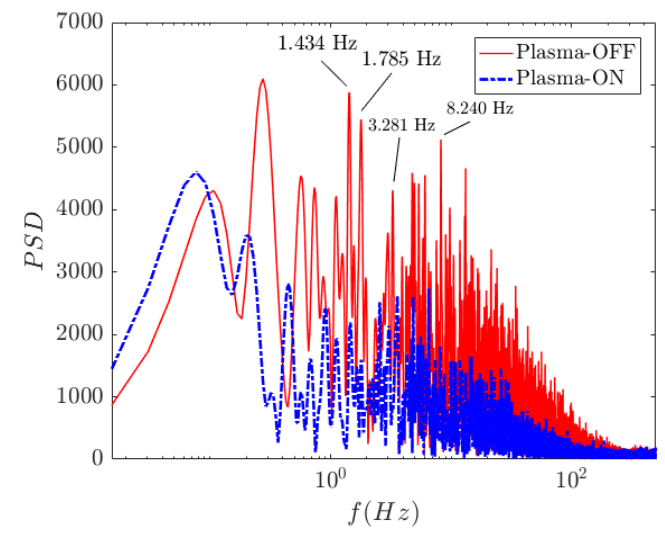

Fig. 15. Frequency spectra of surface pressure signal, $\alpha=23^{\circ}$ and $x / c=0.3$. 
Figures 16-18 show that for the cases of $\alpha>23^{\circ}$ and Plasma-OFF condition several oscillation frequencies by the hot-film sensors are present between the leading-edge and $x / c=0.5$. In these cases, the separated shear layer stands close to the leading-edge and instabilities of the leading-edge shear-layer lead to the vortex shedding and Karman Vortex Street. The order of frequencies of this phenomenon is clearly greater and is reported in the literature to be around 30 to $100 \mathrm{~Hz}$ (Wu et al. 1998; Zaman et al. 1989). Moreover, as it can be seen for Figs. 16-18, these flow structures cannot be measured effectively by the surface shear-stress measurement. It is commonly measured by installation of a hot-wire anemometer one chord upstream of the leading-edge which is not the goal of the present work. The high amplitude frequencies reveal at higher angle of attack near the leadingedge and their sources are not clearly known yet to the author's knowledge. It is hypothesized that they are due to the secondary local separation.

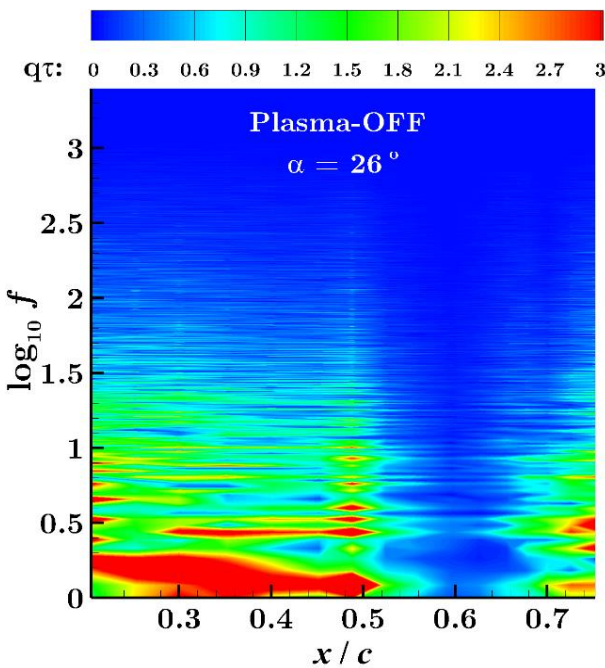

Generally, the current plasma actuator still eliminates the instabilities for angle of attacks greater than $26^{\circ}$, but the actuator is unsuccessful to reattach the flow. Contours of figures 17 and 18 shows this fact. An important point can be concluded when the control authority of the plasma actuator in two cases of near stall and post-stall are compared. When the existing mechanism is the reciprocation of the separation point on the upper surface, the momentum injection has a great flow control performance. However, as the angle of attack is increased to the post-stall region, the imparting momentum will lose its effectiveness. Several studies are performed on the comparison of steady and pulsed operation modes. (Aono et al. 2017, Vernet et al. 2018, Abdollahzadeh et al. 2018 and Messanelli et al. 2020). Abdollahzadeh et al. (2018) examined and compared the flow control authority of the plasma actuation modes.

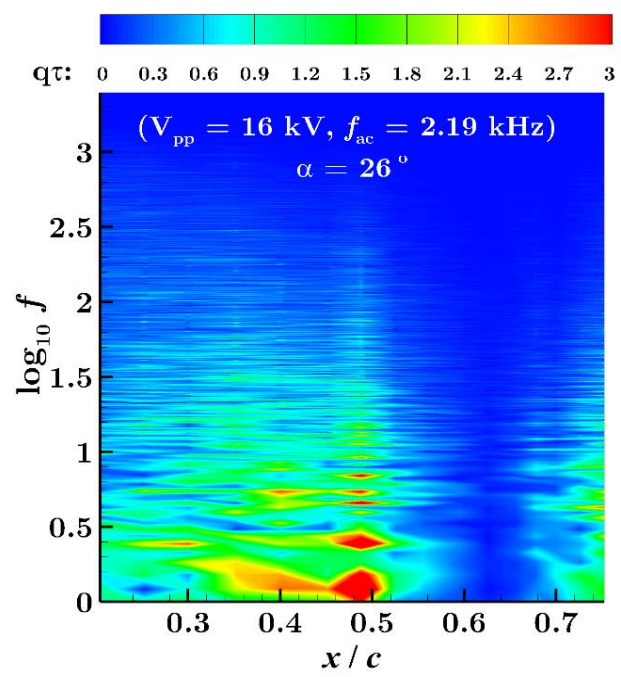

Fig. 16. Space-frequency evolution of quasi-wall shear stress signal: $\alpha=26^{\circ}$.
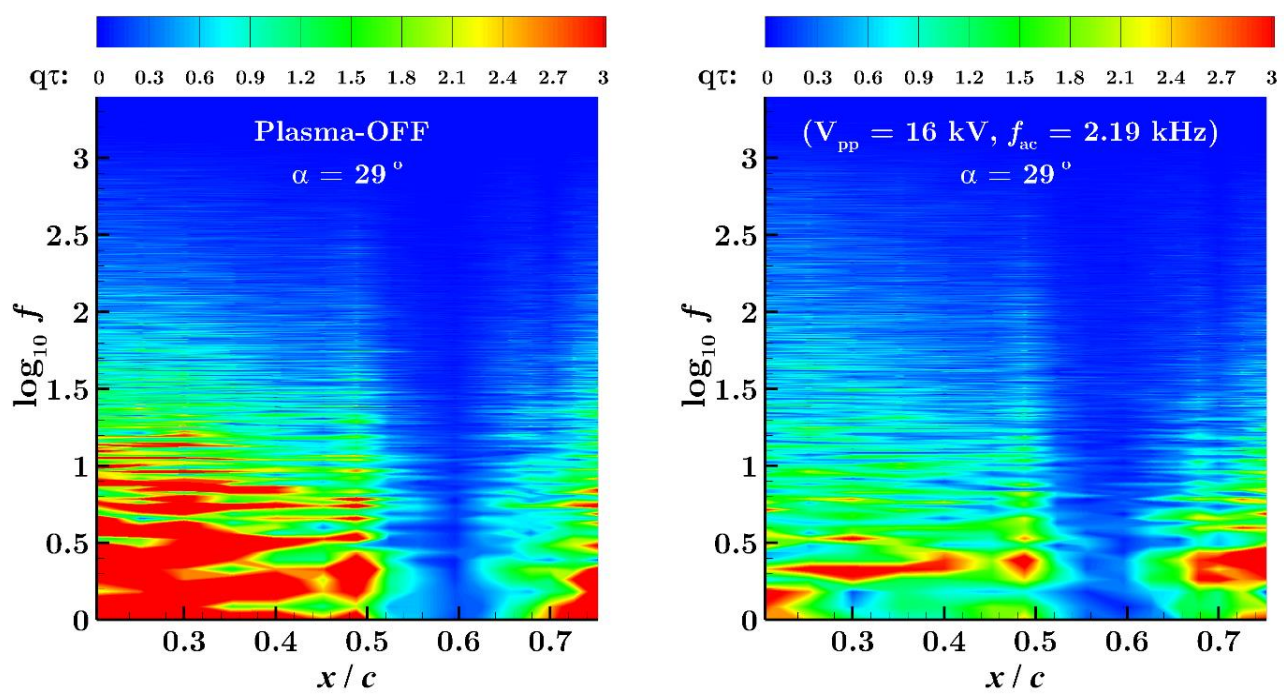

Fig. 17. Space-frequency evolution of quasi-wall shear stress signal: $\alpha=29^{\circ}$. 

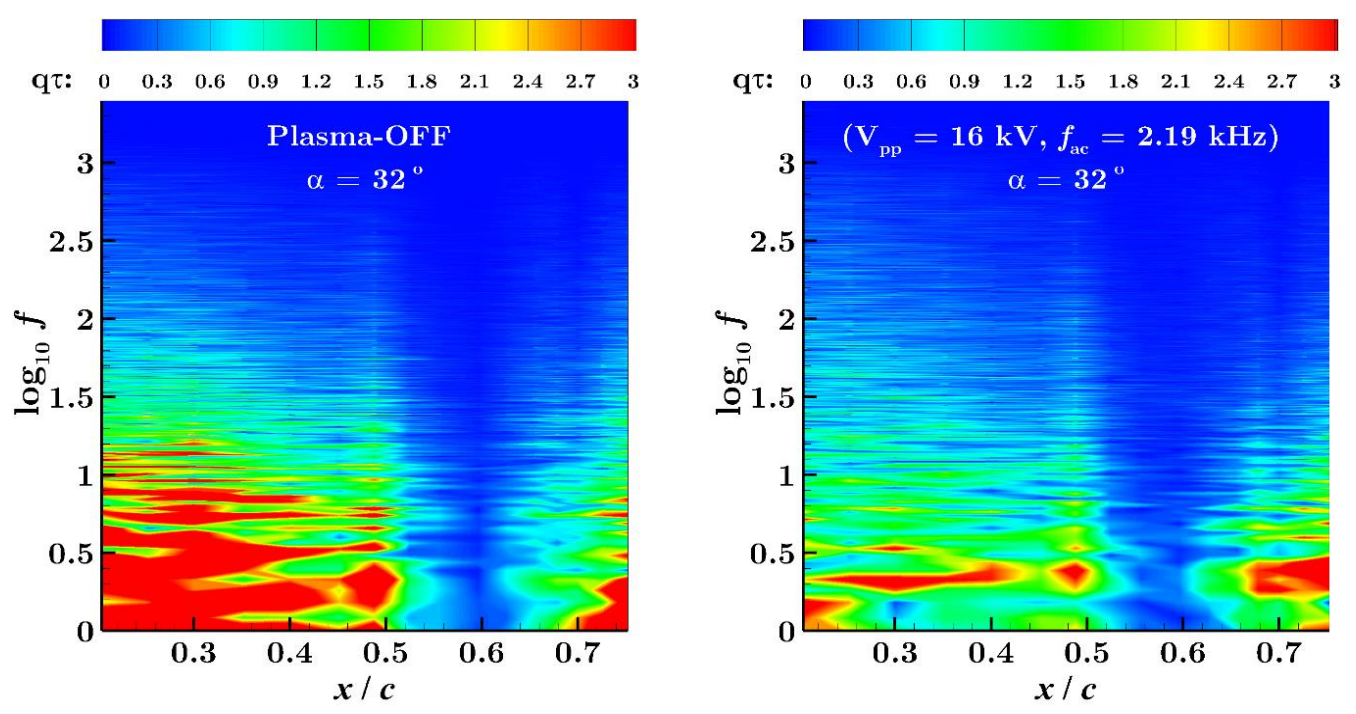

Fig. 18. Space-frequency evolution of quasi-wall shear stress signal: $\alpha=32^{\circ}$.

The use of a plasma actuator in its steady operational mode would cause significant modification of the stability properties in a boundary-layer, and the amplitudes of the oscillations would tend to decrease. On the other hand, in the pulsed actuation mode, the plasma actuator might mitigate or stabilize the natural instabilities of the flow in a more efficient way by generating large coherent vortical structures. In this case, the plasma induced instabilities would counteract the disturbances already present in the flow. Therefore, it is expected that beyond the stall angles of attack of the airfoil, the pulsed actuation mode might be more effective.

\section{CONClusion}

Various flow features of a stalled wind-turbine blade-section are experimentally investigated by surface-mounted hot-film sensors, surface-pressure distribution, and wake-survey studies. All wind tunnel experiments on the blade section were established for a Reynolds number of $0.27 \times 10^{6}$.

The effects of the plasma actuator on the aerodynamics forces are discussed via the liftcoefficient obtained by integrating the surface pressure distribution and the drag coefficient from the wake survey method. The results show an acceptable control authority in the stall region of the airfoil. The deep stall is postponed, and the lift force is recovered for angles of attack beyond the deepstall condition. The drag force is greatly decreased due to the reattachment of the flow and elimination of the vortical structures.

The hot-film sensor's data is discussed and the RMS values as well as the space-frequency spectrum of the quasi-wall shear stress is investigated. Two distinct boundary-layer states are recognized. A state which occurs at the onset and near the deep-stall which is affected by the lowfrequency instabilities of the separated flow. In this case, the steady actuation of the plasma imparts local momentum into the nearby flow and eliminates the instabilities. It will further reattaches the flow over the surface of the airfoil to its unstalled situation. The other state happens beyond the static stall angles of attack of the airfoil in which the flow is fully separated and coexistence of both the leading-edge and the trailing-edge shearlayer instabilities and natural trailing-edge vortex shedding are believed to be the underlying mechanism. In this case, the plasma actuator can eliminate the instabilities to some extent. However, the momentum injection is not effective enough to reattach the flow over the surface of the model. Further experiments using the pulsed actuation mode of the DBD are needed to see whether this actuation mode is able to control the flow at extreme high angles of attack.

\section{REFERENCES}

Abdollahzadeh, M., J. C. Pascoa and P. J. Oliviera (2018). Comparison of DBD Actuators Flow Control Authority in Different modes of actuation. Aerospace Science and Technology 78, 183-196.

Akhlaghi, H., M. R. Soltani and M. J. Maghrebi (2020). Laminar-turbulent intermittency measurement based on the uncalibrated hotfilm data. Measurement 156, No. 107575.

Akhlaghi, H., M. R. Soltani and M. J. Maghrebi (2020). Transitional boundary layer study over an airfoil in combined pitch-plunge motions. Aerospace Science and Technology 98, No. 105694.

Akhlaghi, H., M. R. Soltani and M. J. Maghrebi (2020). Time-Frequency Analysis and Transitional Boundary Layer Investigation over a Pitching Airfoil. Scientia Iranica, Transaction B: Mechanical Engineering 28(2), $860-876$.

Akhlaghi, H. and M. R. Soltani (2020). Comparison of the Transitional Boundary Layer during Pitching and Heaving Motions. Journal of Aircraft, 2020. 
Anderson, J. D. (1991) Fundamentals of Aerodynamics, McGraw Hill, NY.

Aono, H., S. Kawai, T. Nonomura, M. Sato, K. Fujii, K. Okada (2017). Plasma-Actuator Burst-Mode Frequency Effects on LeadingEdge Flow-Separation Control at Reynolds Number 2.6×105. AIAA Journal 55(11), 3789 -3806 .

Armistead Jr., R. A. and J. J. Keyes Jr (1968). Hot Film Sensor for the Study of Wall Turbulence Phenomena. Review of Scientific Instruments, 39(61), 61-68.

Balcon, N., N. Benard, Y. Lagmich, J. P. Boeuf, G. Touchard and E. Morea (2009). Positive and Negative Sawtooth Signals Applied to a DBD Plasma Actuator - Influence on the Electric Wind. Journal of Electrostatics 67, 140-145.

Biganzoli, I., C. Capone, R. Barni and C. Riccardi (2015). Note: Background Oriented Schlieren as a Diagnostics for Airflow control by Plasma Actuators. Review of Scientific Instruments 86(026107), 1-3.

Cattafesta, I. I. I., L. N. and M. Sheplak (2011) Actuators for Active Flow Control. Annual Review of Fluid Mechanics 43, 247-272.

Corke, T. C., M. L. Post and D. M. Orlov (2007). SDBD plasma enhanced aerodynamics: concepts, optimization and applications. Progress in Aerospace Sciences 43(7), 193217.

Duchmann, A., B. Simon, C. Tropea and S. Grundmann (2014). Dielectric Barrier Discharge Plasma Actuators for In-Flight Transition Delay. AIAA Journal 52(2), 358367.

Enloe, C. L., M. G. McHarg and T. E. McLaughlin (2008a). Time-Correlated Force Production Measurements of the Dielectric Barrier Discharge Plasma Aerodynamic Actuator. Journal of Applied Physics 103, 073302, 1-7.

Enloe, C. L., T. McLaughlin, G. I. Font and J. W. Baughn (2006). Parametrization of Temporal Structure in the Single-Dielectric-Barrier Aerodynamic Plasma Actuator. AIAA Journal 44(6), 1127-1136.

Enloe, C. L., G. I. Font, T. E. McLaughlin and D. M. Orlov (2008b). Surface Potential and Longitudinal Electric Field Measurements in the Aerodynamic Plasma Actuator. AIAA Journal 46(11), 2730-2740.

Forte, M., L. Leger, J. Pons, E. Morea and G. Touchard (2006). Plasma Actuators for Airflow Control: Measurement of the nonstationary induced flow velocity. Journal of Electrostatics 63, 929-936.

Gad-el-Hak, M. (1989). Flow Control. applied mechanics reviews 42(10), 261-293.

Greenblatt, D., M. Schulman and A. Ben-Harav (2012). Vertical axis wind turbine performance enhancement using plasma actuators. Renewable Energy 37(1), 345-354.

Guangyin, Z., L. Yinghong, L. Hua, H. Menghu and W. Yun (2015). Flow separation control on swept wing with nanosecond pulse driven DBD plasma actuators. Chinese Journal of Aeronautics 28(2), 368-376.

Hoskinson, A. R., N. Hershkowitz and D. E. Ashpis (2008). Force Measurements of Single and Double Barrier DBD Plasma Actuators in quiescent Air. Journal of Physics D: Applied Physics 41, No. 24. p. 245209.

Jukes, T. N. (2015). Smart control of a horizontal axis wind turbine using dielectric barrier discharge plasma actuators. Renewable Energy 80, 644-654

Jukes, T. N., K. S. Choi, G. A. Johnson, S. J. Scott (2006). Characterization of Surface PlasmaInduced Wall Flows Through Velocity and Temperature Measurements. AIAA Journal. 44(4), 764-771.

Junhui, H., T. C. Corke and F. O. Thomas (2006a). Unsteady Plasma Actuators for Separation Control of Low-Pressure Turbine Blades. AIAA journal 44(7), 1477-1487.

Junhui, H., T. C. Corke and F. O. Thomas (2006b). Plasma Actuators for Separation Control of Low-Pressure Turbine Blades. AIAA journal 44(1), 51-57.

Kriegseis, J., C. Schwarz, A. Duchmann, S Grundmann and C. Tropea (2012). PIV-based Estimation of DBD Plasma-Actuator Force Terms. 50th AIAA Aerospace Sciences, Nashville, Tennessee, AIAA Paper 2012-0411, Jan. 2012.

Labergue, A., L. Leger, E. Moreau and G. Touchard (2006). Effect of a Plasma Actuator on an airflow Along an Inclined Wall: P.I.V. and Wall Pressure Measurements. AIAA Journal 44(4), 764-771.

Little, J., A. Singh, T. Ashcraft and C. Durasiewicz (2018). Post-stall Flow Control using Nanosecond Pulse Driven Dielectric Barrier Discharge Plasma Actuators. Plasma Sources Science and Technology 28, No. 1, p. 014002.

Lombardi, A. J., P. O. Bowles and T. C. Corke (2013). Closed-loop dynamic stall control using a plasma actuator. AIAA journal 51(5), $1130-1141$.

Mahdavi, H., A. Daliri, F. Sohbatzadeh, M. Shirzadi and M. Rezanejad (2020). A single unsteady DBD plasma actuator excited by applying two high voltages simultaneously for flow control. Physics of Plasmas 27. No. 8, p. 083514

McLaughlin, T., M. Munska, J. Vaeth, T. Dauwalter, J. Goode and S. Siegel (2004). Plasma-based actuators for cylinder wake vortex control. $2^{\text {nd }}$ AIAA Flow Control Conference, Portland, Oregon, AIAA Paper 
2004-2129.

Messanelli F., E. Frigerio, E. Tescaroli, M. Belan (2020). Separation Control by Plasma Actuators: Effects of Direct Momentum Injection and Vortex Generation. Flow Turb. Comb. 104, 895 - 926.

Orlov, D., T. Apker, C. He, H. Othman and T. C. Corke (2007). Modeling and experiment of leding edge separation control using SDBD plasma actuators. $45^{\text {th }}$ AIAA Aerospace Sciences Meeting and Exhibit, Aerospace Sciences Meetings, Reno, Nevada, AIAA Paper 2007-877.

Patel, M. P., Z. H. Sowle, T. C. Corke and C. He (2007). Autonomous sensing and control of wing stall using a smart plasma slat. Journal of aircraft 44(2), 516-527.

Porter, C. O., J. W. Baughn, T. E. McLaughlin, C. L. Enloe and G. I. Font (2007) Plasma Actuator Force Measurement. AIAA Journal. 45(7), 1562-1570

Post, M. L. and T. C. Corke (2004a). Separation control on high angle of attack airfoil using plasma actuators. AIAA journal 42(11), 21772184.

Post, M. L. and T. C. Corke (2004b). Separation control using plasma actuators-stationary and oscillating airfoils. 42 $2^{\text {nd }}$ AIAA Aerospace Sciences Meeting and Exhibit, Reno, Nevada, AIAA Paper 0841.

Post, M. L. and T. C. Corke (2006). Separation control using plasma actuators: dynamic stall vortex control on oscillating airfoil. AIAA journal 44(12), 3125-3135.

Pouryoussefi, S. G., M. Mirzaei, F. Alinejad and S. M. Pouryoussefi (2016). Experimental Investigation of Separation Bubble Control on an Iced Airfoil Using Plasma Actuator. Applied Thermal Engineering 100, 13341341.

Rizzetta, D. P. and M. R. Visbal (2007). Numerical Investigation of Plasma-Based Flow Control for transitional Highly-Loaded Low-Pressure Turbine. AIAA journal 42(10), 2554-2564.

Roth, J. R., D. M. Sherman and S. P. Wilkinson (2000). Electrohydrodynamic Flow Control with a Glow-Discharge Surface Plasma. AIAA journal 38(7), 1166-1172.

Saric, W. S., H. L. Reed and E. J. Kerschen (2001). Boundary-Layer Receptivity to Freestream Disturbances. Annual Review of Fluid Mechanics. 34, 291 - 319.

Scime, E., C. Biloiu, C. Compton, F. Doss, D. Venture, J. Heard, E. Choueiri and R. Spektor (2005). Laser induced fluorescence in a pulsed argon plasma. Review of Scientific Instruments 76, 1-3.

Schlichting, H. (1960) Boundary-Layer Theory, McGraw Hill, $4^{\text {th }}$ edition.

Soltani, M. R., A. Daliri, J. Sepahi-Younsi and M. Farahani (2016). Effects of Bleed Position on the Stability of a Supersonic Inlet. Journal of Propulsion and Power 32(5), 1153 - 1166.

Sosa, R. and G. Artana (2006). Steady control of laminar separation over airfoils with plasma sheet actuators. Journal of Electrostatics 64(7), 604-610.

Tathiri, G., H. Parishani, S. G. Pouryoussefi, E. Esmaeilzadeh, S. M. Mirsajedi, M. Mirzaei and N. Khatibzadeh (2016). Experimental Investigation of Separation Control on a NACA0024 Airfoil using Stationary and NonStationary. Journal of Applied Fluid Mechanics 9(2), 877 - 888.

Vernet, J.A., R. Orlu, P.H. Alfredsson (2018). Flow separation control by dielectric barrier discharge plasma actuation via pulsed momentum injection. AIP Advances 8, 075229 (2018), 1 - 19.

Woo, G. T. K. and A. Glezer (2013). Controlled transitory stall on a pitching airfoil using pulsed actuation. Experiments in fluids 54(6), $1-15$.

Wu, J. Z., X. Y. Lu, A. G. Denny, M. Fan and J. M. $\mathrm{Wu}$ (1998). Post-stall flow control on an airfoil by local unsteady forcing. Journal of Fluid Mechanics 371, 21 - 58.

Zaman, K. B. M. Q., D. J. Mckinzi and C. L. Rumsey (1989). A natural low-frequency oscillation of the flow over an airfoil near stalling conditions. Journal of Fluid Mechanics 202, 403 - 442. 